\title{
IP Models to Orchestrate Innovation Ecosystems: IMEC, a PUBLIC RESEARCH INSTITUTE IN NANO-ELECTRONICS
}

\author{
Bart Leten \\ Wim Vanhaverbeke \\ Nadine Roijakkers \\ André Clerix \\ Johan Van Helleputte
}

Companies increasingly organize innovation activities within innovation ecosystems. This study illustrates the central role of the IP-model that an orchestrator develops for the innovation ecosystem partners. The governance of IP is instrumental for the success of innovation ecosystems as it determines the value appropriation potential for the ecosystem partners and positively influences the success of innovation ecosystems. The insights are based on a case study of IMEC, a public research institute in nano-electronics. IMEC has an IP-based orchestration model for innovation ecosystems through multi-party research collaborations between public and private firms. (Keywords: Innovation Management, Intellectual Property, Innovation Networks, Innovation Ecosystems, Open Innovation)

| $\mathrm{n}$ a growing number of industries, the development of new technologies becomes so expensive and risky that companies are forced to join forces in complex innovation networks or ecosystems. A prime example is the semiconductor industry where the costs of developing new generations of semiconductors have increased exponentially. ${ }^{1}$ Depending on the innovation needs of the industry, ecosystems can be made up of different sets of partners at different times where companies collaborate and pool their resources on a temporary basis to achieve joint innovation goals while sharing associated costs and risks. ${ }^{2}$ Innovation ecosystems generate value for partners by reducing development costs and risks and by combining complementary knowledge, enabling partners to address problems with high complexity. ${ }^{3}$ Ecosystem partners can subsequently use the knowledge created within ecosystems to support their own businesses.

While several authors within the ecosystem literature refer to the selforganizing characteristics of ecosystems, other publications stress the role of the leading firm or ecosystem orchestrator in the success of ecosystems. ${ }^{4}$ In fact, authors have pointed out that the particular role the orchestrator plays in shaping the innovation ecosystem, stimulating cooperation amongst partners, setting the research agenda, and adding value through its own capacities can be an important determinant of ecosystem success as well an important source of competitive advantage for 
Bart Leten is Assistant Professor Innovation Management at the Vlerick Business School and the University of Leuven.

Wim Vanhaverbeke is Professor of Strategy and Innovation, BEW Faculty, Hasselt University and Visiting Professor at Esade Business School and National University of Singapore.

Nadine Roijakkers is Assistant Professor of Strategy and Innovation at the Knowledge Center for Entrepreneurship and Innovation (KIZOK) of Hasselt University. André Clerix is Director IP Portfolio and Litigation at IMEC vzw, European and Belgian Patent Attorney, MaNaMa IP law. Johan Van Helleputte is Senior Vice-President Strategic Development at IMEC vzW. the orchestrator. An ecosystem orchestrator can positively influence ecosystem success if it is able to create a structure, including an IP-model, that stimulates cooperation by ensuring value appropriation for all ecosystem partners, and if it is able to keep on attracting partners based on its specific technological expertise. ${ }^{5}$

The purpose of this study is to illustrate the central role of the IP-model that an orchestrator develops to manage and grow an innovation ecosystem. The IP-model determines the value appropriation potential for the ecosystem partners and is in this way crucial in driving successful collaborative innovation initiatives. We study the case of IMEC, ${ }^{6}$ a leading public research institute that orchestrates innovation ecosystems around specific nano-electronics technologies through multi-party research collaborations. These joint research activities are organized in Industrial Affiliation Programs (IAPs), which function on the basis of innovation ecosystems including partners that hold different positions in the semiconductor value chain. The technologies explored within an IAP are costly and have a high risk factor and complexity, ${ }^{7}$ justifying the cost, risk, and talent sharing. To induce companies to collaborate within an IAP, IMEC has designed an IP-model that ensures value appropriation for all partners. Prior research has shown that companies are often unwilling to collaborate if they have ex-ante knowledge appropriation concerns. ${ }^{8}$ These concerns are explicitly addressed by IMEC by negotiating upfront bilateral IP agreements with the ecosystem partners based on an underlying IP-model.

The model is premised on foreground IP, which is developed in the IAPs, and which becomes largely available to all ecosystem partners. Although the goal is to generate generic IP that is of interest to all partners, for each partner there are also possibilities to limit IP sharing and to conduct additional proprietary research with IMEC to acquire exclusively owned IP. The combination of shared and exclusively owned IP allows each partner to build up its own unique IP fingerprint in a cost effective and speedy way. Furthermore, IMEC obtains co-ownership on most foreground IP, which enables it to build up a stronger technological base over time. This puts IMEC in an excellent position to define and initiate new IAPs.

\section{IMEC and the IAPs}

IMEC is headquartered in Leuven ${ }^{9}$, Belgium. It was founded in 1984 by the Flemish government ${ }^{10}$ as a research institute in microelectronics, but it later expanded its research into nano-electronics and applications in chips and systems design, energy, healthcare and life sciences, wireless communication, imaging, and sensor systems. IMEC has grown from €6.5 million in revenues in 1984 to 
$€ 300$ million in 2011. At its foundation, IMEC received more than $90 \%$ of its operating revenues in public grants. This percentage has shrunk to only $15 \%$ in 2011, mainly due to the success of its IAPs. As of 2011, IMEC employed close to 2000 people, including 600 industrial residents, Ph.D. students, and guest researchers. ${ }^{11}$

Research at IMEC is conducted in three phases, i.e. basic, applied, and developmental research. The stage of development of the technology determines the type of collaborations that IMEC undertakes. Basic research is 8-15 years ahead of market applications, and most companies are reluctant to invest in this type of research because of the high degree of uncertainty, long times to market, and value appropriation difficulties. ${ }^{12}$ Basic research is the domain of universities, where researchers examine the basic characteristics of materials and explore different paths to achieve technological goals. IMEC collaborates with over 200 universities attracting Ph.D. students by providing an academic-like environment, access to funds, and state-ofthe-art infrastructure. The research output of Ph.D. theses is selectively patented. IMEC uses the output of its basic research initiatives as background knowledge for initiating new ecosystems thus ensuring that its future orchestration role is grounded in up-to-date technological expertise. ${ }^{13}$

Applied research focuses on technology that is 3-8 years ahead of market needs. It is pre-competitive research, which facilitates collaboration amongst industrial partners. In this phase, IMEC defines and initiates innovation ecosystems by bringing together partners in IAPs to advance research on particular nano-electronics technologies. Over time, IMEC has developed orchestrating capabilities necessary for managing simultaneously a multitude of innovation partners. ${ }^{14}$ As a public research institute, IMEC can be considered as a non-player orchestrator as it is not active in end markets and therefore does not constitute a competitive threat to its partners. This helps IMEC in its role as orchestrator as it creates an environment where partners are willing to openly discuss technology roadmaps enabling IMEC to initiate valuable research programs that correspond to partners' needs.

The concept of IAPs was developed in the early 1990s by J. Van Helleputte, the former Vice President in charge of business development. It is a partnership formula for joint research by industrial researchers and IMEC research teams focused on a specific technology. Within an IAP, actors that typically take different positions in the semiconductor value chain cooperate in a common platform program, which addresses the challenges of applied research in a technical domain. By collaborating in an IAP, companies reduce the costs and risks of applied research. IAP participation also offers companies the option to experiment with alternative technology routes to those followed by in-house applied research. Each industrial partner joins an IAP on the basis of a bilateral contract with IMEC that has a clearly defined technological scope and IP rules. As orchestrator, IMEC ensures the value appropriation potential for all partners, which is an important condition for the success of an innovation ecosystem. ${ }^{15}$

The first two IAPs were launched during the period 1992-1994 and IMEC has coordinated more than 25 IAPs since $2000 .{ }^{16}$ Currently, there are 12 IAPs in operation. Since the beginning of the 1990s, 587 different companies have signed 
contracts with IMEC and participated in at least one IAP. As an example, Exhibit 1 gives a detailed account of the innovation ecosystem (IAP) on 3D systems integration that IMEC orchestrates.

\section{EXHIBIT I. 3D Systems Integration IAP}

The purpose of the 3D systems integration IAP is to conduct collaborative research on a new technology to create electronic circuits (3D integrated chips) which is expected to bring multiple benefits, such as reduced power consumption, new design possibilities, and improved circuit security due to more complex chip designs. IMEC researchers spotted the opportunity of the 3D technology through discussions with universities and companies. Preparation started in 2005 with internal experiments resulting in $\mathbb{P}$ that served as background $I P$ in the $3 D$ ecosystem later on. At that time, IMEC mapped other research experiments conducted elsewhere to identify the most promising technological routes to advance 3D technology. The internal experiments and the mapping resulted in a research program and the set-up of an IAP on 3D systems integration in 2008.

Today, the 3D systems integration IAP brings together IMEC and 34 industrial partners in one innovation ecosystem (see Figure I).

FIGURE I. The 3D Systems Integration Innovation Ecosystem

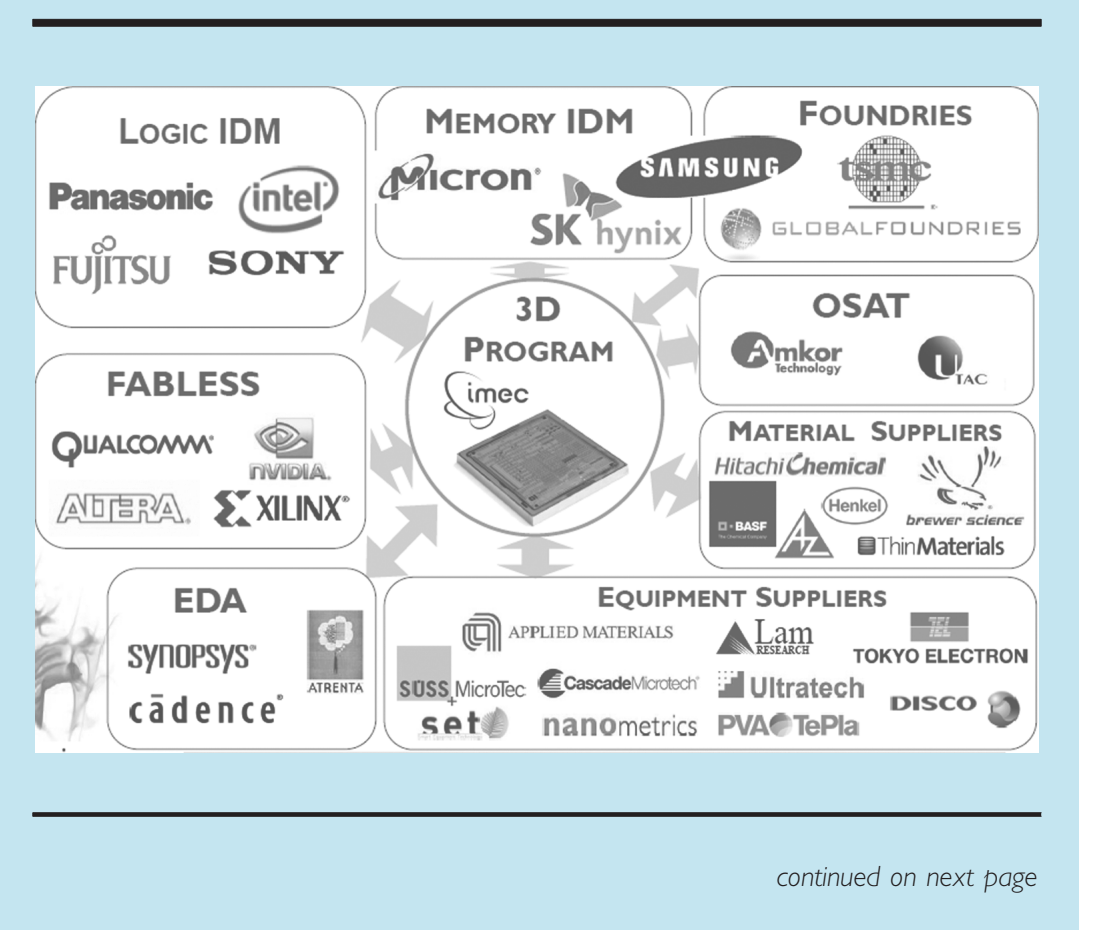


Partners take different positions in the value chain of the nano-electronics industry:

- First, there are the end-users of the 3D technology, such as the fabless companies, Integrated Device Manufacturers (IDMs), and foundries.

- Second, the Electronic Design Automation (EDA) vendors participate in the ecosystem for the development of design software packages.

- Next, the Original Subcontract and Test (OSAT) companies are responsible for the assembly, testing, and packaging of chips.

- Finally, multiple Equipment Suppliers and Material Suppliers develop new types of equipment and materials for manufacturing 3D integrated chips.

At the start of the 3D IAP, IMEC made bilateral IP arrangements with the IAP partners, based on their IP-model and taking into account individual contributions and needs. In general terms, the technology end-users get access to foreground IP related to design and manufacturing. The other ecosystem partners get access to a smaller, more specific set of IP. For example, the equipment suppliers get access to the IP related to their piece(s) of equipment. The equipment and material suppliers typically also negotiated restrictions with respect to the access of others to knowledge on the performance of their specific pieces of equipment and materials. Most of the IAP partners negotiated the possibility to conduct a limited amount of proprietary follow-up research with IMEC on the generic technologies developed in the IAP.

Once the first contracts with key partners were signed, the IAP took off and IMEC researchers began to collaborate with industrial residents on the 3D technology. Other partners entered later on. IMEC has been orchestrating the 3D systems integration IAP for five years now. Only $2 \%$ of the turnover in this ecosystem comes from public funding, 14\% from suppliers, and the remaining $84 \%$ from other partners such as foundries, fabless companies, and IDMs. The IAP has so far resulted in 13 patent filings on the 3D technology and 74 scientific papers. The IP generated in the IAP is used by IAP partners in further internal research and initial production trials. 69\% of the $3 \mathrm{D}$ team is on IMEC's payroll, $24 \%$ are industrial residents from different partners in the IAP and $7 \%$ are $\mathrm{PhD}$ students conducting research in this technological area.

The third research phase is developmental research. This type of research focuses on topics that are 2-3 years ahead of market applications and is based on bilateral collaboration between an IAP partner and IMEC. 


\section{The IP-based Orchestration Model: How IMEC's IP Management Stimulates Collaboration and Progress in IAPs}

\section{Core Principles}

Ownership of and access to the IP-protected knowledge in an IAP is determined beforehand and is part of bilateral agreements between IMEC and its partners. Several principles underlie the IAP IP-model (see Figure 2). First, IMEC's IP that is available at the start of an IAP is labeled "IAP background." It is IMEC's existing IP in science-based research and technologies that is relevant for a new IAP. Second, IP that is generated during the course of an IAP is termed "IAP foreground." Foreground IP consists of all the IP that is generated by IMEC researchers and/or residents of IAP partners at IMEC facilities as a result of the IAP. Upon payment of an entrance fee, IAP partners receive a non-exclusive, non-transferable license necessary for the exploitation of the foreground IP generated within the scope of the IAP. The scope of the license depends on the contributions and technology needs of the different ecosystem partners. ${ }^{17}$ The following foreground IP categories can be distinguished:

- $\mathrm{R}_{0}$-IMEC researchers generate $\mathrm{R}_{0}$ during the course of an IAP without the collaboration of industrial residents. This IP is owned exclusively by IMEC and industrial partners have no (ownership) rights to it. IP access for an IAP partner consists of a non-exclusive, non-transferable license.

- $\mathrm{R}_{1}$-IAP partners generate $\mathrm{R}_{1}$ in collaboration with IMEC researchers. $\mathrm{R}_{1}$ is co-owned ${ }^{18}$ by IMEC and the industrial partner(s) that has (have) contributed to the invention. Each co-owner can use this IP as it wishes. An ecosystem partner can get access to (some of the) IP that IMEC and other partners have developed within the IAP without contributing to it. The access for a non-contributing partner is regulated in the bilateral contract with IMEC and depends on the technological needs of the partner. For example, chip manufacturers need new developments in process technologies, while

FIGURE 2. IMEC's IAP IP-Model

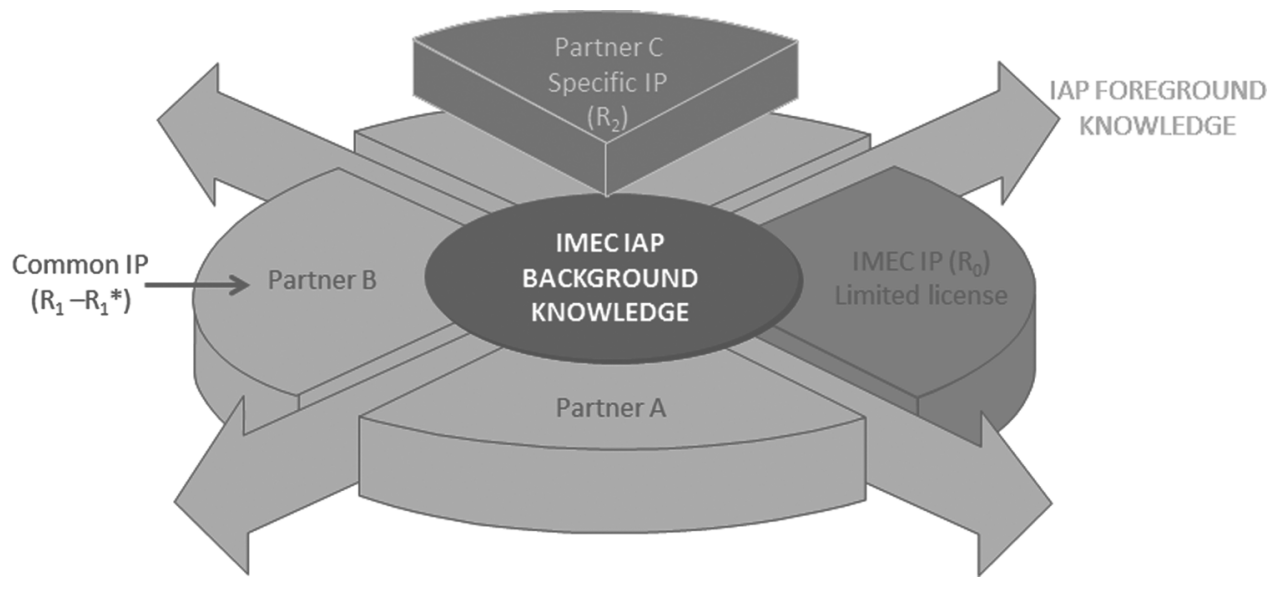


fabless partners that rely on manufacturers to produce their chips, are mainly interested in design and application technologies or the impact of next generation process technologies on their future design strategy. They do not need access to (most of the) manufacturing and process IP. IP access for a non-contributing partner consists of a non-exclusive, non-transferable license. ${ }^{19}$

- $\mathrm{R}_{1}$ *-This IP category refers to knowledge that is not shared among all IAP partners because the partner that (co-)developed the technology does not want to share it with some others (for example, competitors). In this case, restrictions (indicated by the asterisk) are added to the knowledge that can be shared among partners. For example, the purpose of the low-K IAP was to generate knowledge on the operation and efficiency of low-K materials as isolation materials in transistors. Besides chip manufacturers, several providers of equipment to deposit low-K materials on transistors, each using a different material composition, participated in the IAP. Not all partners received access to the full equipment or materials related knowledge that was created. While all partners received access to general knowledge on the operation of low-K materials, knowledge on the performance of specific materials was only shared with the material owning company and not with other equipment companies. The general knowledge is labeled $R_{1}$ and the specific knowledge is categorized as $\mathrm{R}_{1}{ }^{*}$.

- $\mathrm{R}_{2}$-An IAP partner can request to perform limited proprietary research with IMEC researchers in the margin of and in parallel with the IAP. For example, the 3D systems integration IAP (see Exhibit 1) resulted in an IP-protected TSV technology to make an interconnection between chips. To learn more about this technology, some IAP partners asked for additional proprietary $\left(R_{2}\right)$ research to apply the TSV technology to their own processes and wafers. The content and conditions of such research are agreed on upfront between IMEC and the partner. The costs for $\mathrm{R}_{2}$ results are fully borne by the partner and the IP that is generated is exclusively owned by the partner and is not shared with IMEC or others.

\section{Value Appropriation by IAP Partners}

There are different ways through which the above described IP-model set up by IMEC stimulates collaboration and ecosystem progress by allowing partners to appropriate value from their investment and participation in an IAP.

First, IAP partners obtain access to valuable IMEC background IP at an early stage. Background knowledge is scientific information resulting from Ph.D. research and basic research collaborations with academic partners or research conducted by IMEC and its industrial partners in prior IAPs. It is hard to access this knowledge outside the IAP as IMEC only selectively provide licenses on important background technologies.

Second, the majority of the foreground knowledge $\left(R_{0}+R_{1}\right)$ is shared among partners through non-exclusive licensing agreements. In this way, partners obtain access to most of the program outputs while paying for only part of total 
FIGURE 3. IP Fingerprint of IAP Partners

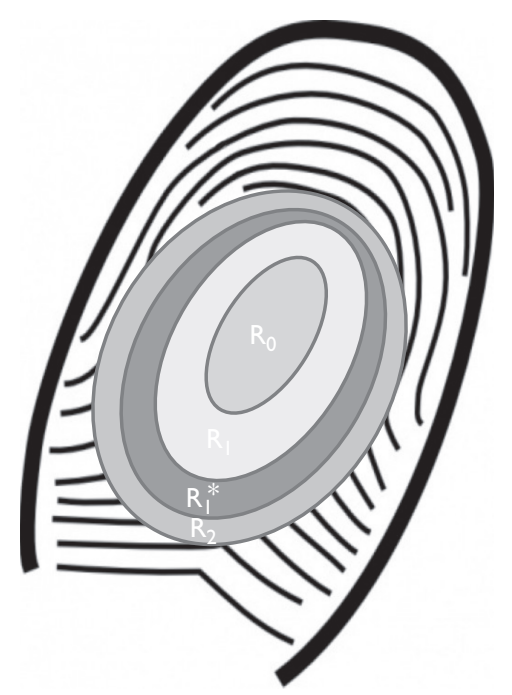

R\&D costs. Sharing R\&D costs has become more important over time as the costs of semiconductor research have soared during the last two decades. Today only the largest companies (such as TSMC, Intel, and Samsung) can afford long-term internal applied research. Through participation in IAPs, semiconductor firms can share costs of long-term applied research and can explore different technological options in cases where there is no clear up-front winner known (yet).

Third, the IP-model allows IAP partners to conduct limited proprietary research to match individual needs $\left(\mathrm{R}_{2}\right)$ and to protect the confidentiality of companyspecific information $\left(\mathrm{R}_{1}{ }^{*}\right)$. In this way, partners can combine generic IAP results with company-specific applications that they develop in parallel with/tangential to the IAP and to which they have exclusive rights. Partners are able to build on the foreground knowledge, combine it with internal knowledge, and improve the quality of their own innovations. ${ }^{20}$ Bilateral contracts between IMEC and each of its partners allow for a high degree of flexibility in IP-modulation (unlike with consortium approaches).

The combination of different types of IP enables IAP partners to build up a unique IP fingerprint in a cost effective and speedy way (see Figure 3). Such an IP fingerprint consists of a mix of background IP, foreground IP that is shared with others $\left(\mathrm{R}_{0}\right.$ and $\left.\mathrm{R}_{1}\right)$, and foreground IP that is not shared with other partners $\left(\mathrm{R}_{1}{ }^{*}\right.$ and $\left.\mathrm{R}_{2}\right)$. This unique IP fingerprint enables IAP partners to differentiate themselves from other companies inside and outside an IAP and to provide ex-post a unique offer to the market hence appropriating economic value.

\section{Value Appropriation by IMEC}

There are different ways through which IMEC appropriates value from orchestrating innovation ecosystems (IAPs). From a static perspective, IMEC appropriates 
value from its IAPs via the program fees that are paid by IAP partners and (Co-) ownership without any accounting on foreground IP $\left(\mathrm{R}_{1}\right)$. From a dynamic perspective, IMEC appropriates value from the orchestration of ecosystems by using the (co-owned) foreground IP of prior IAPs as background knowledge to initiate new IAPs.

Program fees (one-time entrance fee and yearly program fees) compensate IMEC for the background IP that is brought into the IAP, the provision of research facilities and researchers, and the set-up and orchestration of the IAP research programs. ${ }^{21}$ IMEC obtains rights to most of the foreground IP $\left(\mathrm{R}_{1}\right)$ irrespective of its contribution. These rights can be single IP ownership, co-ownership, or a nonexclusive license with sublicensing rights ${ }^{22}$. There are two ways through which IMEC appropriates value from foreground IP. First, and most importantly, IMEC uses such IP as background IP to launch new IAPs. IP from prior IAPs, together with IP from internal basic research, is used dynamically as background IP in new IAPs. Second, IMEC occasionally directly valorizes IP by licensing/transferring/selling technologies and by creating spin-offs. The amount of money generated via direct valorization of IP is limited to 1-2\% of IMEC's revenues.

The choice between direct valorization of IP and safeguarding IP rights for future IAPs is important because the future success of the IP-based orchestration model hinges on the access of IMEC to IP that can be used as background IP in new IAPs. IMEC has thus to decide between direct IP valorization and safeguarding access to IP for its future IAPs: as IMEC first and foremost aims to define, initiate, and orchestrate innovation ecosystems, technologies (and related IP) are only transferred to external companies when they are less relevant for new IAPs or when they are mature and caught up by the market. IMEC will then license-out to manufacturers while safeguarding the IP rights of the IAP partners. Alternatively, IMEC will spin-off a technology when no external entrepreneurs are found to license-in the technology. ${ }^{23}$

\section{Ensuring Future Orchestration Success}

IMEC orchestrates innovation ecosystems in nano-electronics technologies that are pre-competitive in nature. Ecosystem orchestrators should not only focus on current orchestration success, but also seek ways to prolong their orchestration role in the future. In this respect, IMEC is exploring new applications for its IP-based orchestration model, two of which we discuss below.

First, nano-electronics is moving away from a focus on M\&M (More-ofMoore) towards MtM (More-than-Moore). M\&M captures Moore's law and refers to the trend that the number of transistors that can be placed on an integrated circuit doubles approximately every two years, leading to a continuous decrease in costs and increase in performance. The M\&M trajectory becomes increasingly expensive and technologically complex. MtM refers to the practice of adding functionalities on chips (systems on chips or SOCs). This shift poses some challenges for IMEC's IAP IP-model: M\&M is easier to plan via long-term research projects as the industry has a common technology roadmap. MtM pushes research in the direction of more short-term and application-oriented research as market trends are volatile and less predictable. The innovation ecosystem partners will push for less pre-competitive and closer to the market research (less $\mathrm{R}_{1}$ and more $\mathrm{R}_{1}{ }^{*}$ or even $\mathrm{R}_{2}$ ). The IP-based orchestration model (Figure 2) can still be used but IMEC has to find a new balance 
between keeping sufficient IP in common $\left(\mathrm{R}_{1}\right)$ and conducting proprietary research $\left(\mathrm{R}_{2}\right)$ with each partner separately. However, this technological trend should not only be considered as a challenge; it also opens up new opportunities since the nano-electronics value chain becomes even more fragmented and many small, specialized players need an orchestrator to coordinate their innovation activities.

Second, IMEC aims to leverage the IAP model to the life sciences industry in search of nano-electronic applications in this industry. The life science industry is in transition: the pharmaceutical R\&D model is under pressure as the number of newly approved drugs is declining despite increased R\&D spending. The sector faces similar problems as the semiconductor sector in the late eighties when vertically integrated firms could no longer face the technical challenges and costs of RED and gave way to a disintegrated and networked model of technological innovation. Likewise, pharmaceutical companies today are vertically integrated and research is getting more costly and complex and collaboration in innovation ecosystems may be imperative to face these challenges successfully.

IMEC is convinced that their IAP model can be leveraged into life sciences. However, nano-electronics - the expertise of IMEC—will have to be combined with expertise in life sciences. IMEC therefore will team up with a second orchestrator that has strong competences in life sciences to create a dual-core, dual-site innovation ecosystem where two innovation ecosystems are integrated. This is illustrated in Figure 4. In such a system, IMEC and its nano-electronics ecosystem partners will

FIGURE 4. Dual Core-Dual Site Orchestration Model

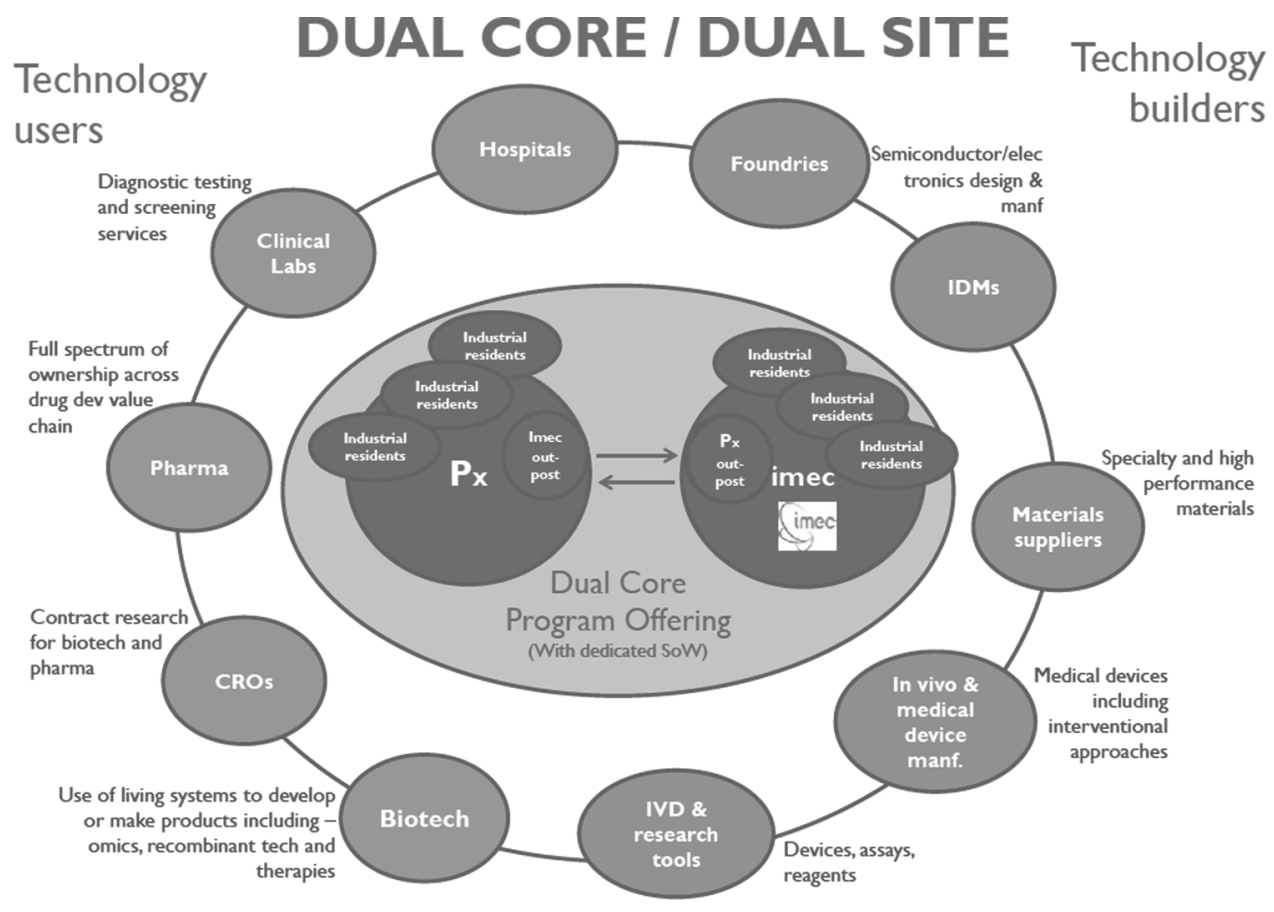


collaborate with a second orchestrator in life sciences and its ecosystem, consisting of hospitals, pharmaceutical companies, clinical labs, CRO's, and biotechnology companies.

The IP rules of the single core IP-model can be largely leveraged to the dual-core model. The IP agreements between IMEC (or the second orchestrator) and its ecosystem partners will remain the same, but additional IP arrangements between both orchestrators are needed. IP ownership will be based on contributions and location. Research developed at IMEC is owned by IMEC and the same holds for the second orchestrator. ${ }^{24}$ Furthermore, contributions of IMEC at the location of the other orchestrator lead to co-ownership by IMEC and vice versa. Finally, there is the possibility to cross-license knowledge for internal use and the right for each orchestrator to grant sub-licenses to its own ecosystem partners, in line with their business, which is assumed not to interfere (substantially) with the rest of the ecosystem.

\section{Conclusions}

IMEC is an interesting example to illustrate how a public firm can successfully orchestrate innovation ecosystems of private firms, what the role of the ecosystem orchestrator is in stimulating success, and how the IP-model is the crux in explaining how an innovation ecosystem can thrive as orchestrator and partners understand how to appropriate value from ecosystem participation. How can an IP-based orchestration model be instrumental in the success of innovation ecosystems? In industries where there is a high need to reduce R\&D costs and risks, the innovation ecosystem orchestrator can stimulate the progress and success of its ecosystems by continuously investing in a strong IP base within its field of expertise and sharing this knowledge with its partners. By giving its partners maximal access to/ co-ownership of the IP created within the innovation ecosystem, the orchestrator enables partners to reap the full benefits of joint research, while they only carry out and pay part of it. Furthermore, a good IP-based orchestration model leaves room for customization. This can be done by offering partners the possibility to conduct proprietary joint research with the orchestrator in parallel with the ecosystem. A successful IP-based orchestration model hinges on two important premises: the needs of partners and their contributions. Through bilateral agreements, the orchestrator has the flexibility to take partner-specific needs into account. Innovation ecosystem orchestrators can prolong their leading role in ecosystems by maintaining a learning organization that is oriented towards building up crucial technological expertise and searching for new ways to apply successful orchestration models.

Innovation ecosystems can also be organized by way of consortia such as SEMATECH ${ }^{25}$ and SGC. ${ }^{26}$ Consortia can achieve important results too, but work in a different, consensus-wise way, without a strong and active orchestrator that determines the direction of the innovation ecosystem, which has both advantages and disadvantages. There are also self-organizing innovation ecosystems where social norms determine to a large extent the functioning of the partners in the ecosystem. Ecosystems in the Dutch vegetables industry offer good examples. ${ }^{27}$ Future research 
may focus on developing a classification scheme of innovation ecosystems with different governance structures and examine the contingencies (including IP-models) under which they can deliver targeted outcomes.

\section{Notes}

1. While early semiconductor fabrication facilities were affordable to many companies, the costs to build an advanced fab moved past $\$ 1$ billion once the features sizes dropped below 180 nano-meters.

2. P.J. Williamson and A. De Meyer, "Ecosystem Advantage: How to Successfully Harness the Power of Partners," California Management Review, 55/1 (Fall 2012): 24-46.

3. S. Nambisan, and M. Sawney, The Global Brain: Your Roadmap for Innovating Faster and Smarter in a Networked World (Upper Saddle River, NJ: Wharton School Publishing/Pearson Press, 2007).

4. G. Lorenzoni and C. Baden-Fuller, "Creating a Strategic Center to Manage a Web of Partners," California Management Review, 37/3 (Spring 1995): 146-163; C. Dhanaraj and A. Parkhe, "Orchestrating Innovation Networks," Academy of Management Review, 31/3 (July 2006): 659-669; S. Nambisan and M. Sawhney, "Orchestration Processes in Network-Centric Innovation: Evidence from the Field," Academy of Management Perspectives, 25/3 (August 2011): 40-57.

5. Ibid. Recent contributions on innovation ecosystems build strongly on the platform literature where the role of the technology leader and the structure this firm puts in place to orchestrate ecosystems are considered as important factors in platform success. A. Gawer and M. Cusumano, Platform Leadership: How Intel, Microsoft, and Cisco Drive Industry Innovation (Boston, MA: Harvard Business School Press, 2002); A. Gawer and M. Cusumano, "How Companies become Platform Leaders," MIT Sloan Management Review, 49/2 (Winter 2008): 29-30; A. Gawer, Platforms, Markets, and Innovation (Cheltenham, UK: Edward Elgar, 2009).

6. Information on IMEC was collected from two sources: Internal company documents; and Interviews with IMEC managers. Data through interviews were collected during the period 2010-2013 starting with exploratory and unstructured interviews providing general information about IMEC's innovation ecosystems and the IP-model applied in the IAPs. Next, we interviewed leading IP and strategy experts at IMEC using an interview guide reflecting the theoretical framework we constructed based on the information from the exploratory interviews. Finally, our understanding of IMEC's ecosystem strategy and IP-model was refined during a few focused interviews during which ambiguities about specific details of the model were clarified with IMEC's IP managers. Each of these interviews were recorded and lasted between one and two hours. We prepared transcripts of the interviews and conducted follow-up phone calls with the interviewees. R. Yin, Case Study Research: Design and Methods (Thousand Oaks, CA: Sage Publications, 2009).

7. Complexity relates in this case to the embryonic nature of the technologies under development, which coincides with uncertainty on the most promising technological routes to advance the technology.

8. B.A. Heiman and J.A. Nickerson, "Empirical Evidence Regarding the Tension between Knowledge Sharing and Knowledge Expropriation in Collaboration," Managerial and Decision Economics, 25/6-7 (September-November 2004): 401-420.

9. Besides Leuven, where the main research labs are located, IMEC has R\&D centers in the Netherlands, India, and Taiwan and representation offices in China, Japan, and the USA.

10. Flanders is the Dutch-speaking region of Belgium where more than $50 \%$ of the population resides.

11. In 2011, IMEC produced 1773 publications, was granted 132 patents, and applied for 133 patents. Since 1984, the research institute has launched 35 spin-offs. In 2011 , IMEC received the prestigious IEEE corporate innovation award for its contribution to CMOS technologies (i.e., scaling research) and its innovative ecosystem orchestration model.

12. R. Nelson, "The Simple Economics of Basic Scientific Research," The Journal of Political Economy, 67/3 (June 1959): 297-306.

13. Gawer and Cusumano (2008), op. cit.

14. Nambisan, and Sawney (2007), op. cit.

15. Nambisan, and Sawhney (2011), op. cit.

16. IAPs typically focus on topics such as high-k dielectrics and metal gates in scaled planar devices, cleaning and contamination control for sub-32 nm process technology, carbon nanotubes 
and semiconducting nanowires, low-k Cu interconnect, photovoltaic energy, body area networks, and 3D systems integration.

17. Several researchers within the ecosystem and platform literature stress that this type of platform-specific knowledge or ecosystem-specific technological expertise contributed by the ecosystem orchestrator is crucial for ecosystem success. Not only does it allow the orchestrator to shape the direction of the ecosystem, but it also enables the orchestrator to repeatedly initiate and design new ecosystems and attract future partners based on its valuable expertise and its dynamically expanding background IP. Gawer and Cusumano (2002), op. cit.

18. The standard rule is that co-ownership takes the form of co-patents. IMEC provides co-owners the freedom to license, but they make agreements on litigation as IMEC wants to limit the number of cases in which they end up as a plaintiff in a litigation lawsuit against a firm that is a partner in one of the IAPs. Collaboration contracts contain litigation clauses that state that patent co-assignees first have to try to reach a mutual agreement with a potential patent infringer before going to trial. When reaching an agreement is not possible and a co-owner initiates an infringement lawsuit, IMEC will mostly take a passive role in which they do not contribute to the costs, and do not share in the potential revenues, from the lawsuits. While co-ownership is the standard rule, IMEC prefers single-ownership coupled to a license with sublicensing rights for their partner and will try to negotiate this. There are two reasons why single-ownership is preferred. First, it gives IMEC more control over their patents. Second, it reduces the patent administration and governance costs.

19. Non-exclusivity guarantees that the results of the IAP are accessible to all partners (in line with their bilateral agreement with IMEC). Non-transferability implies that the licensee cannot assign or otherwise convey the license to any other party beyond the IAP without IMEC and the contributing partners' consent. In this way, the new technology stays within the IAP-ecosystem.

20. B. Cassiman, R. Veugelers, and S. Arts, "How to Capture Value from Linking to Science-Driven Basic Research: Boundary Crossing Inventors and Partnerships," Working Paper, KU Leuven, 2011.

21. IMEC conducts different orchestration tasks within each of the innovation ecosystems (IAPs). First, it defines research programs that are attractive for different types of industrial partners. IMEC understands how to create attractive programs as it takes the bridging role between universities and industry: It understands the firms' technology needs and it stays up-to-date with respect to the latest scientific and technological developments. The drawback is that IMEC is solely responsible for setting up IAPs and consequently fully bears the risks related to establishing new IAPs. Second, IMEC makes bilateral agreements with all IAP partners on the scope of the IAP, deliverables, and IP ownership/access. Bilateral contracts have the advantage that IMEC can quickly start up new IAPs. This contrasts with the consortium approach (e.g., SEMATECH) where decisions are based on a time-consuming consensus model. Third, IMEC coordinates the execution of IAPs. This is done by splitting up the IAP in different technology building blocks, which are executed by a team of researchers and managed by an IMEC employee. The coordination of different building blocks is done by project managers and the IAP scientific director. Finally, IMEC sets up a communication structure to share research findings among partners. IMEC communicates via (bi) weekly meetings with the research teams and via biannual meetings with the senior management of the IAP partners to give an overview of the newly generated IP and to discuss the continuation of the IAP. Partners are selected to attend the meetings depending on the arrangements in the bilateral contracts.

22. The sublicensing rights are necessary for IMEC to guarantee the freedom to use this IP when it sets up new IAPs.

23. IMEC launches spin-offs as follows. First, a feasibility study is conducted and the IP situation is explored. An incubation period of one to two years, during which applications are developed, is necessary to prepare for the spin-off's establishment. Second, IMEC transfers or licenses-out IP to the spin-off. IMEC is actively involved in the development of its spin-offs and since it has its own seed capital it can work quickly, flexibly, and autonomously to establish spin-offs. An example of an IMEC spin-off is EPIGAN, which was established on the basis of IMEC's mature GaN IP for power electronics.

24. The reason is that each orchestrator has a different task and work content.

25. SEMATECH (Semiconductor Manufacturing Technology) started as a consortium of semiconductor manufacturers to develop next-generation manufacturing technology. Today, it has broader industry participation, including IDMs, foundries, fabless, OSATs, equipment, and material suppliers. Member firms participate in pre-competitive research programs on semiconductor technologies, and they get—conditional upon their membership fee-access (licenses) to all the research results of the programs. P. Grindley, D. Mowery, and B. Silverman, "SEMATECH and Collaborative 
Research: Lessons in the Design of High-Technology Consortia," Journal of Policy Analysis and Management, 13/4 (Fall 1994): 723-758; <www.sematech.org>.

26. SGC (Structural Genomics Consortium) is a consortium of pharmaceutical companies, universities, and public and charitable organizations that does research on 3D structures of proteins relevant for human health. The research results are made freely available by publishing and depositing protein structures in an open protein databank. M. Perkmann, "Trading Off Revealing and Appropriating in Drug Discovery: The Role of Trusted Intermediaries," Best Paper Proceedings of the 2009 Academy of Management Meeting, (August 2009).

27. A-P De Man Alliantiebesturing: Samenwerking als precisie-instrument, Koninklijke Van Gorcum/ Stichting Management Studies, (2006), Chapter 7.

California Management Review, Vol. 55, No. 4, pp. 51-64. ISSN 0008-1256, eISSN 2162-8564. () 2013 by The Regents of the University of California. All rights reserved. Request permission to photocopy or reproduce article content at the University of California Press's Rights and Permissions website at http://www.ucpressjournals.com/reprintinfo.asp. DOI: 10.1525/cmr.2013.55.4.51. 
Copyright of California Management Review is the property of California Management Review and its content may not be copied or emailed to multiple sites or posted to a listserv without the copyright holder's express written permission. However, users may print, download, or email articles for individual use. 